\title{
Angiografia Coronariana Minimamente Invasiva Através de Tomografia com Múltiplos Detectores
}

\author{
Minimally Invasive Coronary Angiography Using a Multidetector CT
}

\author{
André Ricardo Vale Rodrigues, Maurício Rezende Barbosa, Marcelo Sá Vieira de Brito, \\ Luciana Costa Silva, Fernando Santana Machado \\ ECOAR Medicina Diagnóstica - Belo Horizonte, MG
}

A trombose coronariana é conhecida como causa de mortes desde o início do século XIX. Tal conhecimento tornou-se a base de uma revolução no tratamento e prevenção do infarto agudo do miocárdio (IAM) no século $X X^{1}$. A cineangiocoronariografia seletiva foi introduzida em 1958, e até há alguns anos era o único exame disponível para estudo anatômico das coronárias, juntamente com a necrópsia. Os exames não-invasivos disponíveis eram voltados para análise da função coronariana, por meio de estresse físico ou farmacológico, numa tentativa de se induzir isquemia miocárdica para depois detectá-la². Com o advento de tomógrafos com múltiplos detectores, estações de trabalho com alta capacidade de processamento e aquisição de imagens com resolução isotrópica, tornou-se possível o estudo anatômico coronariano não-invasivo in vivo com boa acurácia e reprodutibilidade ${ }^{3}$.

A insuficiência coronariana aterosclerótica apresenta dois mecanismos fisiopatológicos distintos, culminando em diferentes entidades clínicas. No primeiro mecanismo, a progressão lenta e gradual de uma placa estável e angiograficamente significativa leva à estenose luminal, com redução do fluxo coronariano distal causando a chamada angina do peito ${ }^{4,5}$. Num segundo mecanismo, a ruptura abrupta de uma placa instável pode induzir uma reação trombótica causando oclusão coronariana quase que instantânea e gerando as chamadas síndromes coronarianas agudas: a angina instável e o infarto agudo do miocárdio (IAM) $)^{6,7}$. Nesse segundo mecanismo, as placas ateroscleróticas são freqüentemente de pequenas dimensões e com grande conteúdo lipídico circundado por uma cobertura fibrosa tênue, daí a sua propensão à ruptura ${ }^{8,9}$. Não raramente passam despercebidas à angiografia invasiva convencional e não produzem quaisquer sintomas até o momento de sua ruptura. Como não causam obstrução luminal significativa, podem não ser detectadas pelos métodos não-invasivos convencionais.

\section{Considerações tÉCNICAs}

As artérias coronárias são vasos de reduzidas dimensões (normalmente com lúmen inferior a 4 $\mathrm{mm}$ ), originando-se da aorta ascendente e distribuindo- se para o miocárdico de maneira a constituir uma complexa estrutura tridimensional. Como estão em íntimo contato com o músculo cardíaco, apresentam intensa motilidade. Tais características tornam a angiografia coronariana minimamente invasiva um esforço magistral, demandando equipamentos de imagem com elevada resolução espacial e temporal ${ }^{10}$.

Em 1998 foram introduzidos tomógrafos com capacidade de aquisição simultânea através de quatro fileiras de detectores e um tempo de rotação mínimo de 500 mseg. Desde então, a estratégia que vem sendo adotada para elevar-se a capacidade imaginológica dos tomógrafos é o aumento do número de seções que são adquiridas simultaneamente. Para tal, foram desenvolvidos equipamentos com quantidades cada vez maiores de fileiras de detectores.

A chave para a aquisição de imagens de boa qualidade reside na capacidade de efetuarem-se "cortes" tomográficos durante a fase do ciclo cardíaco, na qual o movimento das coronárias é mínimo: a telediástole. Para tal, os tomógrafos multislice modernos dispõem de sofisticados algoritmos, capazes de realizar uma "parada cardíaca virtual em diástole". A fase sistólica compreende classicamente o terço inicial do ciclo cardíaco. Na medida em que a freqüência cardíaca aumenta, o tempo da sístole, definido ao ECG como intervalo R-T, tende a sofrer variações mínimas. Por sua vez, a diástole, definida como intervalo T-R, sofre grandes flutuações. A maioria dos laboratórios utiliza betabloqueadores para a manutenção de freqüências cardíacas mais baixas, propiciando imagens de maior clareza e precisão diagnóstica.

Após adequado posicionamento do paciente e punção de acesso venoso periférico, inicia-se a fase de aquisição. Inicialmente é solicitada uma breve apnéia pós-inspiratória, para obtenção do escore de cálcio. Logo após, deverá ser mantida nova apnéia por cerca de 20 segundos, tempo total para obtenção das imagens contrastadas na maioria dos casos. Cabe salientar que o tempo de parada respiratória é variável, sendo superior em indivíduos com tronco ou área cardíaca de grandes dimensões e em portadores de anastomose mamária. Nesse último caso, a necessidade do estudo da origem 
do enxerto leva a cortes com início em porções mais superiores do tórax, prolongando, portando, o período de aquisição. É realizada injeção de contraste iodado (aproximadamente $90 \mathrm{ml}$ ) em uma taxa de infusão de aproximadamente $4 \mathrm{ml} / \mathrm{s}$. Em serviços que dispõem de bomba injetora de duplo lúmen, freqüentemente é realizado um bolus de solução salina a 0,9\% de aproximadamente $40 \mathrm{ml}$, também a uma taxa de infusão de $4 \mathrm{ml} / \mathrm{s}$, logo após a injeção do contraste. A finalidade desse último procedimento é reduzir a opacificação das estruturas cardíacas situadas à direita, permitindo melhor visibilidade da árvore coronariana.

Uma vez adquiridas as imagens, inicia-se o trabalho do cardiologista/imaginologista nas workstations para reconstrução, formatação e interpretação. Há considerável variação dos métodos de manipulação de imagem em razão dos softwares disponibilizados pelos diversos fabricantes, sendo os mais comuns - MPR (multiplanar reformation), MPR oblíquo, MPR curvo, MIP (maximum-intensity projection), luminografia virtual e recontrução tridimensional (3D). Considerando-se a grande possibilidade de ajustes e ângulos de projeção, é fundamental que as imagens sejam manipuladas de maneira que todo o volume do vaso seja visibilizado, tirando-se proveito das vantagens de cada técnica10,11 (figs. 1, 2 e 3).
Embora as imagens em 3D permitam uma boa apreciação da anatomia da árvore coronária em sua distribuição espacial, são as técnicas bidimensionais (cortes longitudinais e axiais) que possibilitam o estudo da placa coronariana com relação a sua dimensão, densidade radiológica e estimativa do comprometimento luminal. Para o estudo da luz coronariana, é de fundamental importância a realização de cortes axiais (transversais) do vaso, tomando-se como referência porções normais adjacentes a lesão (fig. 3). A literatura disponível, comparando as diversas técnicas tomográficas de estimativa do grau de estenose luminal com a angiografia invasiva convencional, fornece resultados variáveis de concordância. Tal fato pode ser explicado, em parte, pela grande variação dos protocolos de análise fornecidos pelos diversos fabricantes.

\section{Aplicabilidade Clínica}

A angiotomografia de coronárias ainda não teve seu papel completamente definido; entretanto, sua capacidade diagnóstica a tem tornado um método cada vez mais indicado na prática clínica diária ${ }^{12,13}$.

A rápida evolução tecnológica que envolve essa nova técnica torna difícil precisar com segurança qual será a real importância do exame na alteração da história da

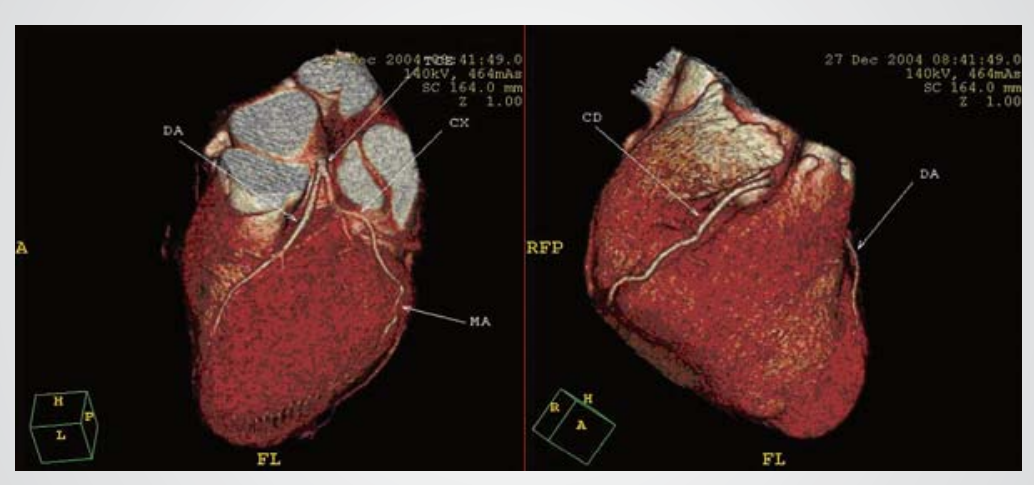

Fig. 1 - Reconstrução tridimensional da árvore coronariana

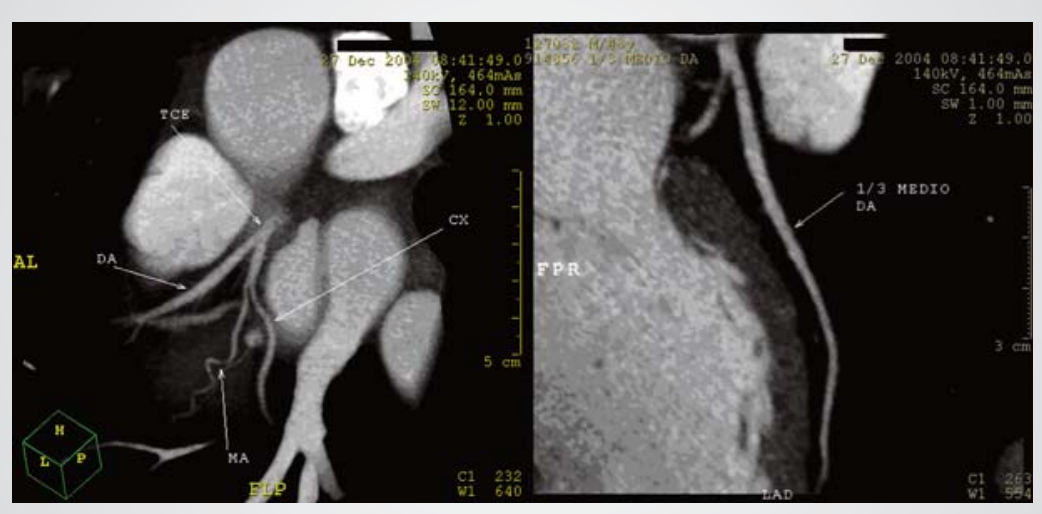

Fig. 2 - Tipos de reconstrução da anatomia coronariana. À esquerda MIP e à direita MIP curvo 


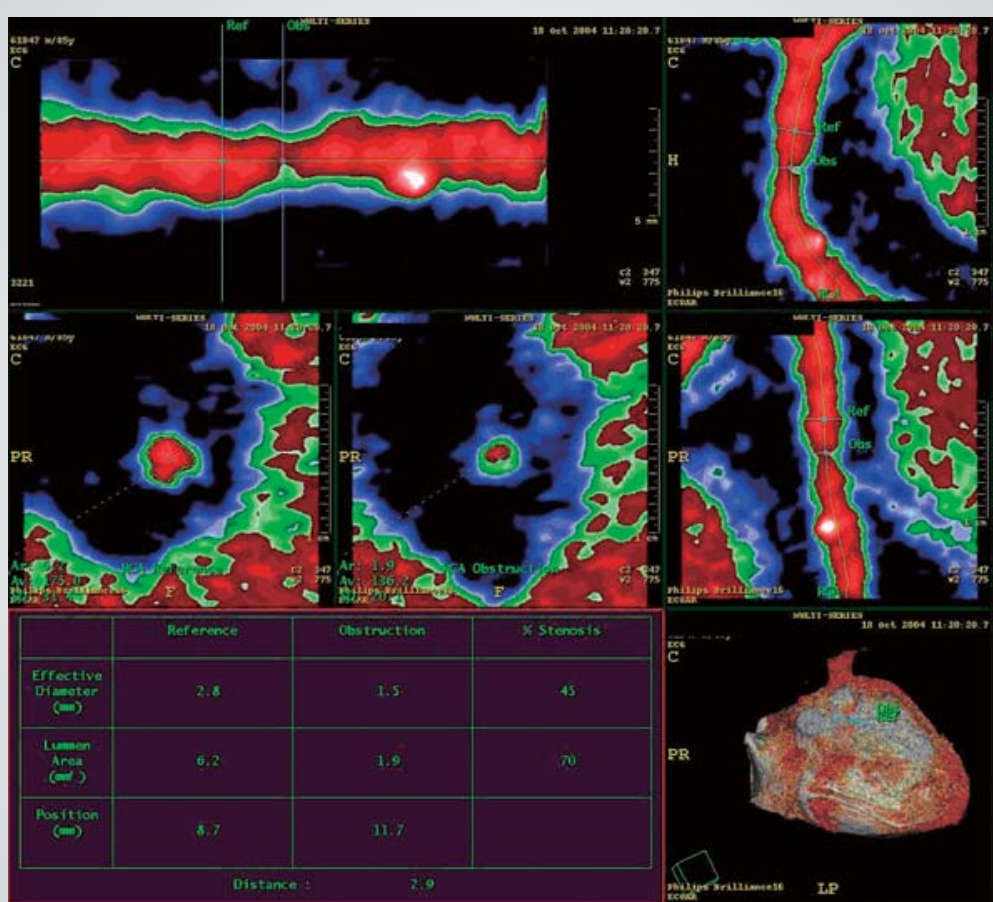

Fig. 3 - Análise de estenose luminal. Contraste intracoronariano em vermelho, com cortes transversais para análise de redução de diâmetro e área

doença coronariana em seu sentido amplo. Da mesma maneira, é importante ressaltar que a angiotomografia coronariana envolve uma metodologia nova e que grandes melhoramentos ainda são necessários e esperados. Entretanto, dados da literatura já apontam para algumas de suas principais indicações, conforme segue.

Em coronárias nativas, alguns autores relataram a capacidade da angio-TC não somente de detectar lesões com boa acurácia, mas também de caracterizar as placas coronarianas de acordo com a sua densidade e conteúdo de cálcio ${ }^{14}$. Schroeder e cols. ${ }^{15}$ correlacionaram a densidade das placas aferidas pela angio-TC contrastada com achados de histologia post-mortem, determinando que placas ricas em lípides possuíam densidade média de $42 \pm 22$ unidades de Hounsfield (HU), placas intermediárias de $70 \pm 21 \mathrm{HU}$, placas calcificadas de $715 \pm 328 \mathrm{HU}$ (fig.4). Infelizmente, a estimativa precisa das características histológicas de uma placa aterosclerótica coronariana mantém-se uma polêmica; Viles-Gonzales e cols. ${ }^{16}$ não encontraram uma relação fidedigna entre a densidade radiológica da lesão, aferida pela angio-TC e os achados histológicos. Novamente, levanta-se a questão da metodologia envolvida na realização do exame e os resultados obtidos.

A técnica de angio-TC tem se mostrado promissora quando aplicada no cenário das síndromes coronarianas agudas. Pacientes portadores de angina instável apresentam placas ateroscleróticas coronarianas cuja densidade é consistentemente inferior àquelas presentes em pacientes com apresentação clínica de angina estável ${ }^{17,18}$. Dorgelo e cols. ${ }^{19}$, estudando pacientes diagnosticados como portadores de síndrome coronariana aguda sem elevação de segmento ST, com tomógrafos de dezesseis fileiras de detectores, obtiveram excelente correlação em comparação à angiografia convencional (sensibilidade de 94\%, especificidade de 96\%). Nessa casuística, 45\% dos pacientes não receberam intervenção percutânea em razão da ausência de obstrução significativa (27\%) ou pela necessidade de revascularização cirúrgica (18\%).

Mollet e cols. ${ }^{20}$ estudaram prospectivamente toda a árvore coronariana de 128 pacientes portadores de angina estável, a fim de se detectar lesões potencialmente passíveis de intervenção, encontrando excelente correlação com a angiografia invasiva (sensibilidade de $92 \%$, especificidade de $95 \%$, valor preditivo positivo de $79 \%$, e valor preditivo negativo de $98 \%$ ).

Pacientes revascularizadados cirurgicamente foram estudados por técnica de tomografia multislice por vários autores ${ }^{21-28}$. A técnica de angio-CT demonstrou-se bastante precisa para avaliação de diversos tipos de anastomoses e pontes vasculares (fig.5), em sítios variados, em pacientes assintomáticos e portadores de sintomas anginosos. Embora os relatos desses diversos autores apontem para um futuro otimista quanto ao estudo anatômico nãoinvasivo dos enxertos vasculares coronarianos, é importante enfatizar que diante de exames tecnicamente inferiores há substanciais fontes de artefatos para erros de interpretação. As anastomoses mamárias, como já mencionado, requerem um tempo superior de apnéia, solicitação freqüentemente difícil de ser atendida em pacientes dementes, de faixa etária elevada e pneumopatas. 


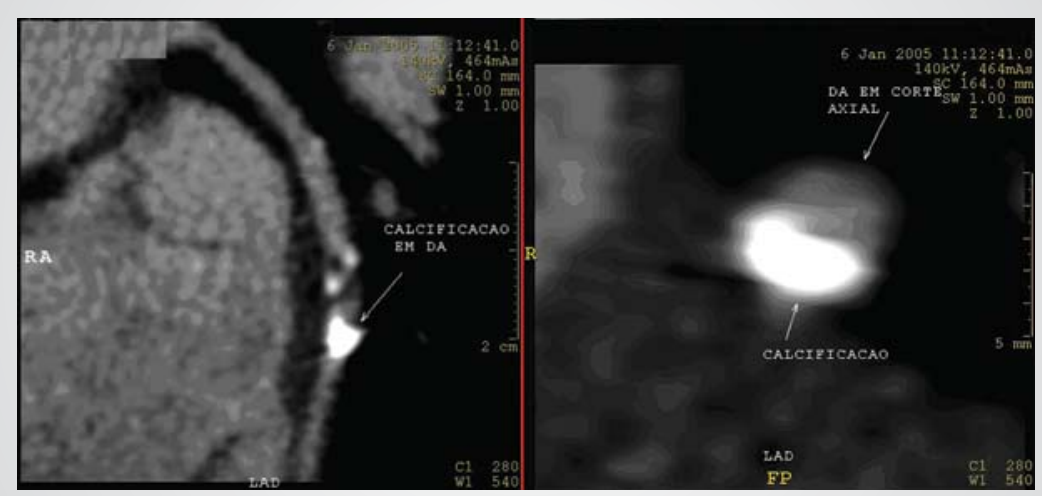

Fig. 4 - Placa mista no terço médio da artéria descendente anterior. À esquerda, observa-se corte longitudinal através de MIP curvo e à direita, corte transversal

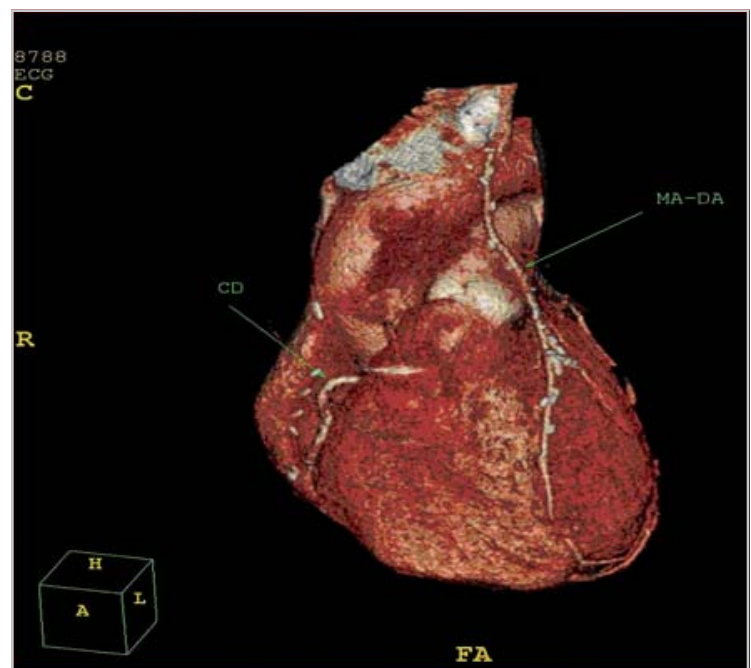

Fig.5 - Anastomose vascular. Presença de anastomose mamária - descendente anterior

A avaliação dos stents por meio de técnicas tomográficas multislice ainda constitui um desafio, na medida em que a elevada densidade de sua malha metálica produz artefatos que reduzem muito a visibilização do contraste intraluminal (fig.6). Entretanto, em alguns casos, o contraste mediante prótese pode ser adequadamente estudado, bem como a sua patência, juntamente com o grau de opacificação arterial distal ${ }^{29-33}$.

Outras aplicações incluem o seguimento do remodelamento arterial coronariano ${ }^{34,35}$, detecção e gradação de aneurismas de coronárias em portadores ou não de arterite de Kawasaki e detecção e caracterização precisa de coronárias anômalas ${ }^{36-39}$.

\section{Cálcio e doença coronariana}

A associação entre a presença de cálcio nas coronárias e placas ateroscleróticas é bem estabelecida e conhecida há quase um século ${ }^{40}$. Em raras situações, pacientes podem apresentar calcificações de Monkenberg na camada média da artéria coronária na ausência de aterosclerose ${ }^{41,42}$. Portadores de alteração do metabolismo do cálcio-fosfato também poderiam apresentar calcificação arterial acelerada ${ }^{43,44}$. Entretanto, para propósitos clínicos, a calcificação coronariana representa uma expressão da doença aterosclerótica ${ }^{45}$.

Os dados obtidos por meio de tomografia por elétrons (EBCT) permitiram a formulação de um escore que descreve a intensidade da calcificação como: (1-10), leve (11-100), moderada (101-399) e acentuada (> 400). A incidência de complicações estaria diretamente relacionada à quantificação fornecida pelo escore, e a ausência de calcificação coronariana detectável tomograficamente possui um elevado valor preditivo negativo em se tratando da exclusão de processo aterosclerótico significativo, sendo, portanto, um achado bastante útil, por exemplo, em uma população portadora de precordialgia atípica ${ }^{45}$. Meneghelo e cols. ${ }^{46}$ descrevem uma distribuição de escores de cálcio determinados por EBCT em 2.253 homens brasileiros, de 22 a 88 anos, encontrando escores superiores a zero em $48,8 \%$ dos casos, numa distribuição que não segue a curva de Gauss.

Trabalhos recentes têm demonstrado que a tomografia multislice é, no mínimo, comparável à $\mathrm{EBCT}^{47,48}$, sendo essa última pouco disponível em nosso meio, em razão do elevado custo e da reduzida versatilidade. Como a maioria dos dados prognósticos foi obtida com base em estudos prospectivos utilizando-se a EBCT, a comparação entre os métodos permanece motivo de controvérsia.

Nenhum dos estudos disponíveis até o momento sobre o valor preditivo dos níveis de calcificação coronariana é baseado em estudos prospectivos rigorosos e concluídos. Assim sendo, o real impacto do chamado escore de cálcio ainda precisa ser demonstrado em grandes coortes populacionais.

\section{ACURÁCIA diagnóstica}

Em se tratando de estudos de acurácia diagnóstica em angio-CT, é de fundamental importância ressaltar a dificuldade para sua comparação e interpretação, na medida em que técnicas distintas são empregadas. Uma análise mais minuciosa dos trabalhos revela diversidade nas seguintes variáveis: 


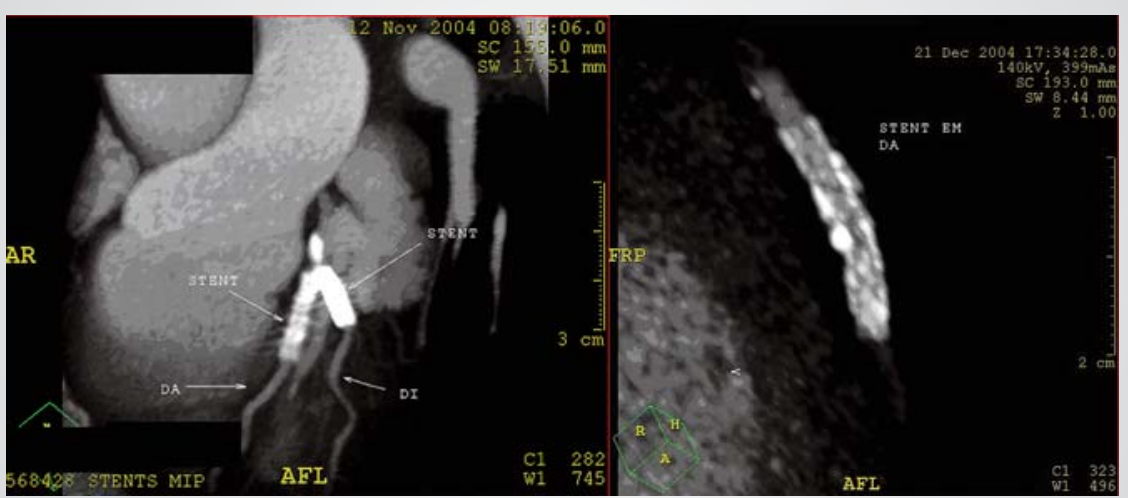

Fig.6 - Stents. À esquerda, observa-se a presença de dois stents cuja malha metálica prejudica a observação luminal. À direita, a presença de contraste dentro do stent pode ser apreciada

- Algoritmos de reconstrução e fases do ciclo cardíaco reconstruídas;

- Paciente estudado;

- Tipo, volume e fluxo de solução de contraste injetado;

- Fabricante dos tomógrafos;

- Software utilizado para interpretação.

O exame para visibilização não-invasiva das coronárias requer equipamento de alta resolução, tanto temporal como espacial. Desde os relatos iniciais no ano $2000^{49,50}$, vários autores têm contribuído para a análise da acurária na detecção de estenose coronariana, estando demonstrada a tendência de superioridade dos tomógrafos equipados com dezesseis fileiras de detectores já nos trabalhos preliminares, em relação aos seus predecessores de quatro fileiras de detectores (tab. 1).

Enxertos cirúrgicos (safena, mamária e artéria radial) têm menor movimentação durante o ciclo cardíaco comparativamente às coronárias nativas, sendo a técnica bem indicada para seguimento de pacientes revascularizados (tab.2).
Com o surgimento de tomógrafos multislice mais modernos, englobando tecnologias de 32, 40 e 64 detectores $^{60}$, espera-se uma elevação ainda maior na qualidade das imagens e, naturalmente, da acurácia diagnóstica.

\section{LIMITAÇÕES}

Como previamente mencionado, a calcificação coronariana tem sido considerada um marcador de doença aterosclerótica. Entretanto, segmentos intensamente calcificados têm a avaliação luminal prejudicada pela produção de artefatos na luz arterial, à semelhança do que ocorre em portadores de stents.

Para aquisição de imagens de boa qualidade é de todo desejável a presença de ritmo cardíaco com intervalo $\mathrm{R}-\mathrm{R}$ regular e freqüências cardíacas mais baixas ${ }^{61,62}$, e a maioria dos laboratórios tem utilizado betabloqueadores para obtenção de freqüências cardíacas inferiores a 65 $\mathrm{BPM}^{63}$. Naturalmente, a presença de arritmias cardíacas,

Tabela 1 - Acurácia da angio-TC coronariana utilizando-se tomógrafos de quatro e dezesseis fileiras de detectores: estudos preliminares com dezesseis fileiras de detectores

\begin{tabular}{|c|c|c|c|c|c|}
\hline Autor & $n$ & Detectores & Sensib. & Especif. & Não aval. \\
\hline Nieman et al ${ }^{51}$ & 31 & 4 & $81 \%$ & $97 \%$ & $27 \%$ \\
\hline Achenbach et $a^{52}$ & 64 & 4 & $91 \%$ & $84 \%$ & $32 \%$ \\
\hline Knez et al ${ }^{53}$ & 42 & 4 & $78 \%$ & $98 \%$ & $6 \%$ \\
\hline Herzog et $a^{54}$ & 42 & 4 & $72 \%$ & $92 \%$ & - \\
\hline Kopp et $a^{55}$ & 102 & 4 & $86-93 \%$ & $96-97 \%$ & $18 \%$ \\
\hline Becker et al ${ }^{56}$ & 28 & 4 & $78 \%$ & $71 \%$ & $11 \%$ \\
\hline Nieman et al ${ }^{57}$ & 53 & 4 & $82 \%$ & $93 \%$ & $30 \%$ \\
\hline Nieman et $\mathrm{al}^{3}$ & 59 & 16 & $95 \%$ & $86 \%$ & $0 \%$ \\
\hline Ropers et $\mathrm{al}^{58}$ & 77 & 16 & $92 \%$ & $93 \%$ & $12 \%$ \\
\hline
\end{tabular}

Tabela 2 - Acurácia da angio-TC na avaliação de pontes e anastomoses coronarianas

\begin{tabular}{|c|c|c|c|c|}
\hline Autor & n pacientes & $\mathrm{n}$ anastom. & Sensib. & Especif. \\
\hline Burgstahler et al ${ }^{21}$ & 10 & 21 & $86 \%$ & $100 \%$ \\
\hline Ko et $a^{23}$ & 39 & 115 & $93,3 \%$ & $99 \%$ \\
\hline Tello et $\mathrm{al}^{22}$ & 26 & 75 & $97 \%$ & $95 \%$ \\
\hline Schlosser et $\mathrm{al}^{26}$ & 48 & 131 & $96 \%$ & $95 \%$ \\
\hline Rossi et al ${ }^{59}$ & 47 & 116 & $100 \%$ & $100 \%$ \\
\hline
\end{tabular}


sobretudo as taquiarritmias com R-R variável, como no caso da fibrilação atrial, torna freqüentemente impraticável a realização do exame. Batimentos ectópicos, mesmo isolados, podem prejudicar a adequada visibilização de segmentos coronarianos ${ }^{64}$ (fig. 7).

A dose de radiação para realização da angiotomografia coronariana é considerada elevada e superior àquelas utilizadas na tomografia por emissão de elétrons e na angiografia convencional ${ }^{65}$ (tab. 3).

Como na angiografia clássica, o exame demanda injeção venosa de contraste iodado, com potencial efeito nefrotóxico e alergênico.

\section{Conclusão}

No início do século XX, o estudo da anatomia das artérias coronárias era praticado em mesas de necrópsia. Na segunda metade do século, a cineangiocoronariografia propiciou um salto gigantesco na abordagem da doença aterosclerótica coronariana. A partir do ano de 2000, iniciou-se o estudo anatômico minimamente invasivo das coronárias por meio de tomógrafos equipados com múltiplos detectores.
Podem-se citar como principais qualidades da técnica a rapidez, a segurança e a boa acurácia diagnóstica, principalmente em se tratando de valores preditivos negativos. As maiores limitações são constituídas por arritmias durante a aquisição das imagens, dificuldade do paciente para realizar apnéia, calcificação coronariana extensa e presença de stents.

As principais indicações para utilização da técnica apontam para o diagnóstico das anomalias anatômicas coronarianas, alternativa em pacientes portadores de teste ergométrico duvidoso, dor precordial atípica, baixo escore de cálcio, acompanhamento de portadores de pontes e anastomoses vasculares coronarianas, portadores de miocardiopatia e eventualmente em avaliações pré-operatórias.

Atualmente, a angiotomografia com múltiplos detectores encontra-se em franco desenvolvimento. No momento, a qualidade de imagem oferecida não é robusta o suficiente para fazer do método uma ferramenta de rotina para todos os pacientes ${ }^{67,68}$. A capacidade de se qualificar o tipo de placa visibilizada traz novas perspectivas na avaliação do risco coronariano; entretanto, investigações nessa área são necessárias.

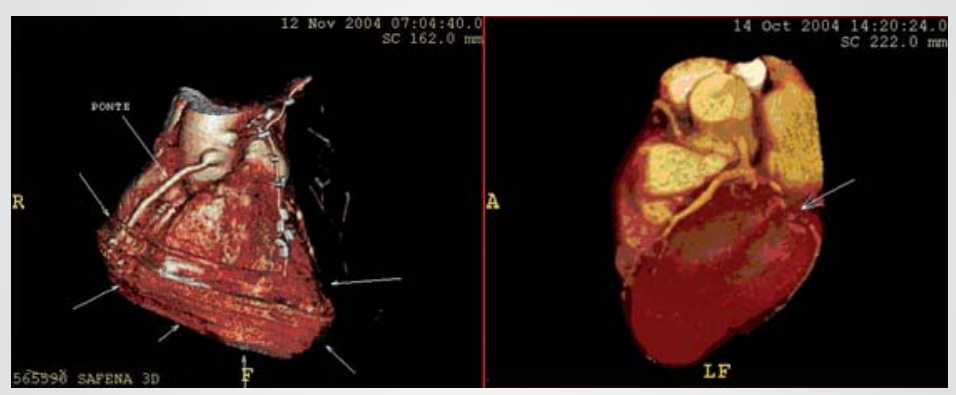

Fig.7 - Artefatos de imagem. Imagem à esquerda demonstrando "degraus" produzidos por respiração durante a fase de aquisição. À direita, observa-se artefato no terço proximal da artéria circunflexa devido a ectopias ventriculares

Tabela 3 - Doses de radiação durante a realização da angio CT

\begin{tabular}{|c|c|c|c|c|c|}
\hline $\begin{array}{l}\text { Dose } \\
\text { Órgão (mSv) }\end{array}$ & EBCT & Siemens ${ }^{\circledR}$ & $\begin{array}{l}\text { Schroder } \\
\text { et al }\end{array}$ & $\begin{array}{c}\text { Achenbach } \\
\text { et } a^{50,52}\end{array}$ & $\begin{array}{c}\text { Angiografia } \\
\text { invasiva }\end{array}$ \\
\hline Medula óssea & 2,0 & 14,1 & 11,0 & 10,1 & 2,5 \\
\hline Pulmões & 3,9 & 37,6 & 27,9 & 22,7 & 8,1 \\
\hline Tireóide & 0,4 & 2,7 & 3,4 & 1,4 & 0,5 \\
\hline Esôfago & 3,1 & 27,1 & 16,9 & 17,2 & 4,2 \\
\hline Fígado & 1,8 & 13,5 & 9,8 & 9,9 & 2,9 \\
\hline Estômago & 2,6 & 18,2 & 9,8 & 9,8 & 1,6 \\
\hline Cólon & 1,0 & 1,0 & 0,7 & 0,9 & 0,7 \\
\hline Bexiga & 0,3 & 0,4 & 0,3 & 0,3 & 0,3 \\
\hline Mamas & 5,9 & 44,0 & 28,7 & 25,6 & 6,9 \\
\hline Ovários & 1,5 & 0,6 & 1,4 & 0,9 & 1,1 \\
\hline Testículos & 0,4 & 0,7 & 0,4 & 0,2 & 0,5 \\
\hline Dose efetiva (mSv) & $0,5-2,0$ & $10,9-13,0$ & $7,6-9,2$ & $6,7-8,1$ & $2,1-2,5$ \\
\hline
\end{tabular}




\section{REFERÊNCIAS}

1. Sarmento-Leite R, Krepsky AM, Gottsschall CAM. Infarto agudo do miocárdio: um século de história. Arq Bras Cardiol 2001; 77: 592601.

2. Rittman EL. Cardiac computed tomography imaging: a history and some future possibilities. Cardiol Clin 2003; 21:491-513.

3. Nieman K, Cademartiri F, Lemos PA, Raaijmakers R, Pattynama PM, Feyter PJ. Reliable noninvasive coronary angiography with fast submillimeter multislice spiral computed tomography. Circulation 2002; 106:2036-8

4. Hangartner J, Charleston A, Davies MI, Thomas AC. Morphological characteristics of a clinically significant coronary artery stenosis in stable angina. Br Heart J 1986; 56:501-8.

5. Roberts W. The coronary arteries and left ventricle in clinically isolated angina pectoris. Circulation 1976; 54:388-90.

6. Mann J, Davies N. Vulnerable plaque relation of characteristics to degree of stenosis in human coronary arteries. Circulation 1996; 94:928-31

7. Davies M, Woolf N, Robertson W. Pathology of acute myocardial infarction with particular reference to occlusive coronary thrombi. $\mathrm{Br}$ Heart J 1976; 38:659-64

8. Ross R. The pathogenesis of atherosclerosis a perspective for the 1990s. Nature 1993; 362:801-9.

9. Davies MJ. The composition of coronary artery plaques. N Engl J Med 1997; 336: 1312-4.

10. Van Ooijen PM, Ho KY, Dorgelo J, Oudkerk M. Coronary artery imaging with multidetector CT: visualization issues. Radiographics 2003; 23:16.

11. Schroder S, Kopp AF, Ohnesorge B. Virtual coronary angioscopy using multislice computed tomography. Heart 2002; 87:195-7.

12. Vignaux O, Paul JF, Duboc D. Multislice CT and MRI of coronary artery disease: current and future role. J Radiol 004; 85:1786-95.

13. Achenbach S, Daniel WG. Noninvasive coronary angiography - an aceptable alternative? N Eng J Med 2002; 345:1909-10.

14. Achenbach S, Moselewski F, Ropers D, Ferencik M, Hoffmann U, MacNeil $B$ et al. Detection of calcified and noncalcified coronary atherosclerotic plaque by contrast-enhanced submillimeter multidetector spiral computed tomography: a segment-based comparison with intravascular ultrasound. Circulation 2004; 109:14-7.

15. Schroeder S, Kuettner A, Leitritz M , Janzen J, Kopp AF, Herdeg C et al. Reliability of differentiating human coronary plaque morphology using contrast-enhanced multislice spiral computed tomography: a comparison with histology. J Comput Assist Tomogr 2004; 28:449-54

16. Viles-Gonzalez JF, Michael Poon, Javier Sanz J, Rius T, Nikalaou K, Fayad ZA et al. In Vivo 16-slice, multidetector-row computed tomography for the assessment of experimental atherosclerosis: comparison with magnetic resonance imaging and histopathology . Circulation 2004; 110:1467-72

17. Inoue F, Sato Y, Matsumoto N, Tani S, Uchiyama T. Evaluation of plaque texture by means of multislice computed tomography in patients with acute coronary syndrome and stable angina. Circ J 2004; 68:840-4.

18. Caussin C, Othanessian A, Lancelin B, Rachal S, Hennequin R, Dambrin $\mathrm{G}$ et al. Coronary plaque burden detected by multislice computed tomography after acute myocardial infarction with near normal coronary arteries by angiography. Am J Cardiol 2003; 92:849-52.

19. Dorgelo J, Willems TP, Geluk CA, van Ooijen PM, Zijlstra F, Oudkerk M. Multidetector computed tomography-guided treatment strategy in patients with non-ST elevation acute coronary syndromes: a pilot study. Eur Radiol 2004;15:708-13.
20. Mollet NR, Cademartiri F, Nieman K, Saia F, Lemos PA, McFadden EP et al. Multislice computed coronary angiography in patients with stable angina pectoris. J Am Coll Cardiol 2004; 43:2265-70.

21. Burgstahler C, Kuettner A, Kopp AF, Herdez C, Martensen J, Claussen $C D$ et al. Non invasive evaluation of coronary artery bypass grafts using multi-slice computed tomography; initial clinical experience. Int J Cardiol 2003; 90:275-80.

22. Tello R, Hartnell GG, Costello P, Ecker CP. Coronary artery bypass graft flow: qualitative evaluation with cine single-detector row $\mathrm{CT}$ and comparison with findings at angiography. Radiology 2002; 224::913-8.

23. Ko YG, Choi DH, Jang YS, Chung NS, Shim WH, Cho SY, Yoo KJ. Assessment of coronary artery bypass graft patency by multislice computed tomography. Yonsei Med J 2003; 44:438-44

24. Kham MF, Herzog C, Landerberger K, Maataouri A, Martens S, Akermann $\mathrm{H}$ et al. Visualisation of non-invasive coronary bypass grafts: 4-row vs. 16-row multidetector computed tomography. Eur Radiol 2005; 15:118-26

25. Yoo KJ, Choi D, Choi BW, Lim SH, Chang BC. The comparison of the graft patency after coronary artery bypass grafting using coronary angiography and multi-slice computed tomography. Eur J Cardiothorac Surg 2003; 24:86-91.

26. Schlosser T, Konorza T, Hunold P, Kuhl H, Schmermund A, Barkhausen J. Noninvasive visualization of coronary artery bypass grafts using 16-detector row computed tomography. J Am Coll Cardiol 2004; 44:1224-9

27. Willmann JK, Weishaupt D, Kobza R, Verdun FR, Seifert B, Marincek $B$ et al Coronary artery bypass grafts: ECG-gated multi-detector row CT angiography - influence of image reconstruction interval on graft visibility. Radiology 2004;232:568-77

28. Dewey M, Lembcke A, Enzweiller C, Hamm B, Rogalla P. Isotropic half-millimeter angiography of coronary artery bypass with 16 slice computed tomography. Ann Thorac Surg 2004; 77:800-4.

29. Kruger S, Mahnken AH, Sinha AM, Borghans A, Dedden K, Hoffmann R et al. Multislice spiral computed tomography for the detection of coronary stent restenosis and patency. Int J Cardiol 2003; 89:167-72.

30. Schuijf JD, Bax JJ, Jukema JW, Lamb HJ, Warda HM, Vliegen HW et al. Feasibility of assessment of coronary stent patency using 16-slice computed tomography. Am J Cardiol 2004; 94:427-30

31. Hong C, Chrysant GS, Woodart PK, Bae KT. Coronary artery stent patency assessment with in-stent contrast enhancement measured at multi-detector row CT angiography: initial experience. Radiology 2004; 233:286-91

32. Nieman K, Cademartiri F, Raaijmakers R, Pattynama P, de Feyter P. Noninvasive angiographic evaluation of coronary stents with multislica spiral CT. Herz 2003; 28:136-42.

33. Ligabue G, Rossi R, Ratti C, Ligabue G, Romagnoli R, Modena MG. Noninvasive evaluation of coronary artery stents patency after PTCA. Radiol Med 2004; 108:128-37.

34. Schoenhegen P, Tuzcu EM, Stillman AE, Moliterno DJ, Halliburton SS, Kuzmiak AS et al. Non-invasive assessment of plaque morphology and remodeling in mildly stenotic coronary segments: comparison of 16-slice computed tomography and intravascular ultra-sound. Coron Artery Dis 2003; 14:459-62.

35. Imazeke T, Sato Y, Inoue F, Anazawa T, Tani S, Matsumoto N et al. Evaluation of coronary artery remodeling in patients with acute coronary syndrome and stable angina by multislice computed tomography. Circ J 2004; 68:1045-50.

36. Sohn S, Kim HS, Lee SW. Multidetetctor row computed tomography for follow-up of patients with coronary artery aneurysms due to Kawasaki disease. Pediatr Cardiol 2004; 25:35-9. 
37. Sato $\mathrm{Y}$, Kato M, Inoue F, Fukui T, Imazeki T, Mitsui M et al. Detection of coronary artery aneurysms, stenoses and occlusions by multislice spiral computed tomography in adolescents with Kawasaki disease. Circ J 2003, 67:427-30.

38. Shi H, Aschoff AJ, Brambs HJ, Hoffmann MH. Multislice CT imaging of anomalous coronary arteries. Eur Radiol 2004;14;2172-81.

39. Deibler AR, Kuzo RS, Vohringer M, Page EE,Safoord RE, Patron JN et al. Imaging of congenital coronary anomalies with multislice computed tomography. Mayo Clin Proc 2004; 79:1017-23.

40. Faber A. Die Arteriosklerose, ihre pathologische Anatomie, ihre Pathogenese und Aetiologie. G Fischer 1912.

41. Mönckenberg JG. Mediaverkalkung und atherosklerose. Virchows Arch Pathl Anat 1914; 216:408-16.

42. Lachman AS, Spray TL, Kerwin DM, Shugoll GI, Roberts WC. Medial calcification of Monkenberg. Am J Med 1977; 63:615-22.

43. Oh J, Wunsch R, Turzer M, Bahner M, Raggi P, Querfeld U et al. Advanced coronary and carotid arteriopathy in young adults with childhood-onset chronic renal failure. Circulation 2002; 106:100-5.

44. Raggi P, Boulay A, Chasan-Taber S, Amin N, Dillon M, Burke SK et al. Cardiac calcification in adult hemodialysis patients. A link between end-stage renal disease and cardiovascular disease? J Am Coll Cardiol 2002; 39:695-701.

45. O'Rourke LA, Brundage BH, Froelicher VF, Greenland P, Grundy SM, Hachamovitch R et al. ACC/AHA Expert consensus document on electron-beam computed tomography for the diagnosis and prognosis of coronary artery disease. J Am Coll Cardiol 2000; 36:32-40.

46. Meneghelo RS, Santos RD, Almeida B, Hiddal J, Martinez T, Moron R et al. Distribuição dos escores de cálcio coronariano determinados pela tomografia ultra-rápida em 2253 homens brancos assintomáticos. Arq Bras Cardiol 2003; 81(supl. 7):27-31.

47. Stanford W, Thompson BH, Burns TL, Heery SD, Burr MC. Coronary artery calcium quantification at multi-detector row helical CT versus electron-beam CT. Radiology 2004; 230:397-402.

48. Hong C, Bae KT, Pilgram TK. Coronary artery accuracy and reproducibility of measures with multi-detector row CT- assessment of effects of different thresholds and quantification methods. Radiology 2003; 227:795-801.

49. Ohnesorge B, Flohr T, Becker C, Kopp AF, Schoepf UJ, Baum U et al. Cardiac imaging by means of electrocardiographically gated multisection spiral CT: initial experience. Radiology 2000; 217:564-71

50. Achenbach S, Ulzheimer S, Baum U, Kachebriess M, Ropers D, Giesler $T$ et al. Noninvasive coronary angiography by retrospectively ECG-gated multislice spiral CT. Circulation 2000; 102:2823-8.

51. Nieman K, Oudkerk M, Rensing BJ, van Ooijen P, Munns A, van Geuns RJ et al. Coronary angiography with multi-slice computed tomography. Lancet 2001; 357:599-603.

52. Achenbach S, Giesler T, Ropers D, Ulzheimer S, Derlien H, Schulte $C$ et al. Detection of coronary artery stenoses by contrast- enhanced, retrospectively ECG-gated, multi-slice spiral CT. Circulation 2001; 103:2535-8

53. Knez A, Becker CR, Leber A, Ohnesorge B, Becker A, White C et al. Usefulness os multislice spiral computed tomography angiography for determination of coronary artery stenoses. Am J Cardiol 2001; 88:1191-4.
54. Herzog C, Abolmaali N, Balzer JO, Baunach AS, Ackermann H, Dogan $S$. Heart-rate adapted image reconstruction in multidetector row cardiac $\mathrm{CT}$ : influence of physiological and technical pré-requisite on image quality. Eur Radiol 2002; 12:1670-8.

55. Kopp AF, Schroder S, Kuettner A, Baumbach A, Georg C, Kuzzo R et al. Non-invasive coronary angiography with high resolution multidetectorrow computed tomography. Results in 102 patients. Eur Heart J 2002; 23:1714-25.

56. Becker CR, Knez A, Leber A, Treede H, Ohnesorge B, Schoeph UJ et al. Detection of coronary artery stenoses with multislice helical CT angiography. J Comp Assist Tomogr 2002; 26:250-5

57. Nieman K, Rensing BJ, van Geuns RJ, Munne A, Ligthart JM, Pattynama PM et al. Usefulness of multislice computed tomography for detecting obstrutive coronary artery disease. Am J Cardiol 2002; 89:913-8.

58. Ropers D, Baum U, Pohle K, Anders K, Ulzhmer S, Ohnesorge B et al. Detection of coronary artery stenoses with thin-slice multi detector row spiral computed Tomography and planar Reconstruction. Circulation 2003; 107:664-6.

59. Rossi R, Chiurlia E, Ratti C, Ligabue G, Romagnoli R, Modena MG. Noninvasive assessment of coronary artery bypass graft patency by multislice computed tomography. Ital Heart J 2004; 5:36-41.

60. Leschka S, Alkadhi H, Plass A, Desbiolles L, Grunenfelder J, Marincek B et al. Accuracy of MSCT coronary angiography with 64-slice technology: first experience. Eur Heart J 2005;26:1482-7.

61. Mennicke M, Giesler T, Ropers D, Baum U, Ulzheimer S, Wenkel E et al. Influence of heart rate in image quality and detection of coronary stenoses with multislice spiral CT. Biomed Tech 2002; 47(Suppl 1): 782-5.

62. Schroder S, Kopp AF, Kuettner A, Burgstahler S, Herdeg C. Influence of heart rate on vessel visibility in noninvasive coronary angiography using new multislice computed tomography: experience in 94 patients. Clin Imaging 2002; 26:106-11

63. Nieman K, Rensing BJ, van Geuns RJ, Vos J, Pattynama PM, Krestin GP et al. Non-invasive coronary angiography with multislice spiral computed tomography: impact of heart rate. Heart 2002; 88:470-4.

64. Choi HS, Choi BW, Choe KO, Choi D, Yoo KJ, Kim MI et al. Pitfalls, artifacts, and remedies in multi-detector row CT coronary angiography. Radiographics 2004; 24:787-900.

65. Schroeder S, Kopp AF, Baumbach A, Kuettner A, Herdeg C, Rosenberger $A$ et al. Noninvasive detection of coronary lesions by multislice computed tomography: results of the new age pilot trial. Catheter Cardiovasc Interv 2001; 53:352-8.

66. Schroeder S, Kopp AF, Baumbach A, Kuettner A, Georg C, Ohnesorge $B$ et al. Non-invasive characterisation of coronary lesion morphology by multi-slice computed tomography: a promising new technology for risk stratification of patients with coronary artery disease. Heart 2001; 85:576-8.

67. Achenbach S. Clinical use of multi-slice CT coronary angiography. Herz 2003; 28:119-25.

68. Achenbach S, Ropers D, Pohle K, Anders K, Baum U, Hoffmann U et al. Clinical results of minimally invasive coronary angiography using computed tomography. Cardiol Clin 2003; 21:549-59. 